\title{
Microbiology Susceptibility Modified Reported Name
}

National Cancer Institute

\section{Source}

National Cancer Institute. Microbiology Susceptibility Modified Reported Name. NCI

Thesaurus. Code C162148.

An indication or description of a change in a microbiology susceptibility agent. 